\title{
Early bond strength of two resin cements to Y-TZP ceramic using MPS or MPS/4-META silanes
}

\author{
Özcan, M ; Cura, C ; Valandro, L F
}

\begin{abstract}
For cementation of yttrium-stabilized tetragonal zirconium polycrystal (Y-TZP) ceramic frameworks, protocols of surface-conditioning methods and available cements vary, resulting in confusion among clinicians regarding selection and effects of different conditioning methods on cement adhesion. This study evaluated the effect of two silanes (3-trimethoxysilylpropylmethacrylate (MPS) and 3-trimethoxysilylpropylmethacrylate/4methacryloyloxyethyl trimellitate anhydride methyl methacrylate (MPS/4-META) on the adhesion of two resinbased cements (SuperBond and Panavia F 2.0) to Y-TZP ceramic and compared several protocols with those indicated by the manufacturer of each of these cements. Disks of Y-TZP ceramic (LAVA, 3M ESPE) $(n=60)$ were divided into six experimental groups ( $\mathrm{n}=10$ per group) and treated as follows: (1) silica coating (SC) + MPS silane + SuperBond; (2) SC + MPS/4-META + silane + SuperBond); (3) SC + MPS silane + Panavia F 2.0); (4) SC + MPS/4META silane + Panavia F 2.0); (5) no conditioning + MPS/4-META silane + Super-Bond (SuperBond instructions); and (6) $50-\mu \mathrm{m} \mathrm{Al}(2) \mathrm{O}(3)$ conditioning + Panavia F 2.0 (Panavia F 2.0 instructions). The specimens were subjected to shear-bond testing after water storage at $37^{\circ} \mathrm{C}$ for 3 months in the dark. Data were analyzed by analysis of variance and Tukey's HSD $(\alpha=0.05)$. After silica coating, the mean bond strength of SuperBond cement was not significantly different between MPS and MPS/4-META silanes (20.2 \pm 3.7 and $20.9 \pm 1.6 \mathrm{MPa}$, respectively), but the mean bond strength of Panavia F 2.0 was significantly higher with MPS silane (24.4 $\pm 5.3 \mathrm{MPa})$ than with MPS/4-META $(12.3 \pm 1.4 \mathrm{MPa})(\mathrm{P}<0.001)$. The SuperBond manufacturer's instructions alone resulted in significantly higher bond strength $(9.7 \pm 3.1 \mathrm{MPa})$ than the Panavia F 2.0 manufacturer's instruction $(0 \mathrm{MPa})(\mathrm{P}<$ 0.001 ). When silica coating and silanization were used, both SuperBond and Panavia F 2.0 cements demonstrated higher bond strengths they did when the manufacturers' instructions were followed. With SuperBond, use of MPS or MPS/4-META silane resulted in no significant difference when the ceramic surface was silica coated, but with Panavia F 2.0, use of MPS silane resulted in a significantly higher bond strength than use of MPS/4-META. Use of chairside silica coating and silanization to condition the zirconia surface improved adhesion compared with the manufacturers' cementation protocols for SuperBond and Panavia F 2.0 resin cements.
\end{abstract}

DOI: https://doi.org/10.1007/s10266-010-0144-1

Posted at the Zurich Open Repository and Archive, University of Zurich

ZORA URL: https://doi.org/10.5167/uzh-58533

Journal Article

Published Version

Originally published at:

Özcan, M; Cura, C; Valandro, L F (2011). Early bond strength of two resin cements to Y-TZP ceramic using MPS or MPS/4-META silanes. Odontology, 99(1):62-7.

DOI: https://doi.org/10.1007/s10266-010-0144-1 


\section{Mutlu Özcan • Cenk Cura • Luiz Felipe Valandro \\ Early bond strength of two resin cements to Y-TZP ceramic using MPS or MPS/4-META silanes}

\begin{abstract}
For cementation of yttrium-stabilized tetragonal zirconium polycrystal (Y-TZP) ceramic frameworks, protocols of surface-conditioning methods and available cements vary, resulting in confusion among clinicians regarding selection and effects of different conditioning methods on cement adhesion. This study evaluated the effect of two silanes (3-trimethoxysilylpropylmethacrylate (MPS) and 3-trimethoxysilylpropylmethacrylate/4-methacryloyloxyethyl trimellitate anhydride methyl methacrylate (MPS/ 4-META) on the adhesion of two resin-based cements (SuperBond and Panavia F 2.0) to Y-TZP ceramic and compared several protocols with those indicated by the manufacturer of each of these cements. Disks of Y-TZP ceramic (LAVA, 3M ESPE) $(n=60)$ were divided into six experimental groups ( $n=10$ per group) and treated as follows: (1) silica coating (SC) + MPS silane + SuperBond; (2) SC + MPS/4-META + silane + SuperBond); (3) SC + MPS silane + Panavia F 2.0); (4) SC + MPS/4-META silane + Panavia F 2.0); (5) no conditioning + MPS/4-META silane + SuperBond (SuperBond instructions); and (6) $50-\mu \mathrm{m} \mathrm{Al}_{2} \mathrm{O}_{3}$ conditioning + Panavia F 2.0 (Panavia F 2.0 instructions). The specimens were subjected to shear-bond testing after water storage at $37^{\circ} \mathrm{C}$ for 3 months in the dark. Data were analyzed by analysis of variance and Tukey's HSD $(\alpha=0.05)$. After silica coating, the mean bond strength of SuperBond cement was not significantly different between MPS and
\end{abstract}

\footnotetext{
M. Özcan $(\varangle)$

Dental Materials Unit, Center for Dental and Oral Medicine Clinic for Fixed and Removable Prosthodontics and Dental Materials Science, University of Zürich, Plattenstrasse 11, CH-8032, Zürich, Switzerland

Tel. +41-44-634560; Fax +41-44-6344305

e-mail: mutluozcan@hotmail.com

C. Cura

Department of Prosthodontics, School of Dentistry, Ege University, Izmir, Turkey
}

\section{L.F. Valandro}

Division of Prosthodontics, Department of Restorative Dentistry, School of Dentistry, Federal University of Santa Maria, Santa Maria, Brazil
MPS/4-META silanes $(20.2 \pm 3.7$ and $20.9 \pm 1.6 \mathrm{MPa}$, respectively), but the mean bond strength of Panavia F 2.0 was significantly higher with MPS silane $(24.4 \pm 5.3 \mathrm{MPa})$ than with MPS/4-META $(12.3 \pm 1.4 \mathrm{MPa})(P<0.001)$. The SuperBond manufacturer's instructions alone resulted in significantly higher bond strength $(9.7 \pm 3.1 \mathrm{MPa})$ than the Panavia F 2.0 manufacturer's instruction $(0 \mathrm{MPa})(P<$ 0.001 ). When silica coating and silanization were used, both SuperBond and Panavia F 2.0 cements demonstrated higher bond strengths they did when the manufacturers' instructions were followed. With SuperBond, use of MPS or MPS/4-META silane resulted in no significant difference when the ceramic surface was silica coated, but with Panavia F 2.0, use of MPS silane resulted in a significantly higher bond strength than use of MPS/4-META. Use of chairside silica coating and silanization to condition the zirconia surface improved adhesion compared with the manufacturers' cementation protocols for SuperBond and Panavia F 2.0 resin cements.

Key words Adhesion - Resin cement - Silane coupling agents $\cdot$ Surface conditioning $\cdot$ Zirconia

\section{Introduction}

The introduction of yttrium tetragonal zirconia polycrystal (Y-TZP) (hereafter, zirconia)-based ceramics in dentistry have made metal-free fixed dental prosthesis (FDP) possible for both anterior and posterior restorations. ${ }^{1,2}$ Yttrium oxide is added to pure zirconia to stabilize it at room temperature and to generate a multiphase material known as partially stabilized zirconia. This type of ceramic material has high initial strength $(2000 \mathrm{~N})$ and fracture toughness (9-10 $\left.\mathrm{MPa} / \mathrm{m}^{2}\right)$, almost twice the values obtained with alumina-based materials, and almost three times those demonstrated by lithium disilicate-based ceramics. ${ }^{1}$ Tensile stresses acting at the crack tip induce a transformation of the metastable tetragonal zirconium oxide form to a monoclinic form. This transformation is associated with a local increase of $3 \%-5 \%$ in volume. This increase in volume results in 
localized compressive stresses being generated around and at the tip of the crack, counteracting the external tensile stresses acting on the fracture tip. This physical property is known as transformation toughening, and it gives zirconiabased ceramics physical and mechanical advantages over glassy matrix ceramics. ${ }^{3-5}$

The only problem related to the performance of such ceramics is that adhesion of resin cements to them is inferior compared to adhesion to silica-based ceramics. ${ }^{6,7}$ The zirconia ceramic has a glass-free polycrystal microstructure making it an acid-resistant or non-etchable material. ${ }^{1,6}$ Some manufacturers and researchers have tried to modify the surface properties of zirconia by means of various airborne-particle abrasion methods followed by silanization. ${ }^{711}$ This approach has been recently criticized as possibly causing subcritical crack growth within zirconia. ${ }^{12}$ Another suggested method is tribochemical silica coating and silanization, which is recommended for the adhesion of resin cements to high-strength ceramics. ${ }^{7-10}$ In this technique, the surfaces are air-abraded with aluminum trioxide particles coated with silica. The blasting pressure results in the embedding of silica-coated alumina particles in the ceramic surface, rendering the silica-modified surface chemically more reactive to the resin when combined with silane coupling agents. Silanes, hybrid organic-inorganic compounds, can function as mediators and promote adhesion between dissimilar inorganic and organic matrices through dual reactivity. ${ }^{13}$ Silane molecules react with water to form silanol groups (-Si-OH) from their methoxy groups (-Si-O$\mathrm{CH}_{3}$ ). The silanol groups then react further with the silica surface to form a siloxane (-Si-O-Si-O-) network. Monomeric ends of the silane molecules react with the methacrylate groups of the adhesive resins by a free radical polymerization process. According to the latest theories, a hydrophobic and branched polysiloxane film that may also contain free hydrogen-bonded oligomers, is formed. ${ }^{14}$ The most commonly applied silane in dental laboratories and chairside is a monofunctional $\gamma$-methacryloxypropyltrimethoxysilane or 3-trimethoxysilylpropylmethacrylate (MPS). MPS is used to optimize and promote adhesion through chemical and physical coupling between metal-, ceramic-, and composite resin. Any composite resin material that contains methacrylate groups in its composition can be used in conjunction with MPS silanes. ${ }^{15}$ Another type of silane coupling agent, MPS/4-META, also contains a diffusion promoting monomer, 4-methacryloyloxyethyl trimellitate anhydride (4-META).

For ceramics exhibiting similar surface compositions and chemical states of silicon and oxygen, such as is attained by the embedding of silica, then it is reasonable to hypothesize that a siloxane bond will be achieved, because the embedded silica provides binding sites for the coupling agent to the ceramic surface. While there is still no consensus in the dental literature as to which surface conditioning method is most suitable for improved adhesion of resin cement to zirconia ceramics, ${ }^{7-11}$ manufacturers of some cements do not suggest any additional surface conditioning to such ceramics.

Potential cements for use with zirconia are based on bisphenol glycydil methacrylate (bis-GMA), 4-META (with methyl methacrylate, 4-META/MMA), or 10-methacryloxydecyldihydrogen phosphate (MDP) monomers. One of the most frequently used dual polymerized, filled adhesive resin cements in dentistry is Panavia F 2.0 (Kuraray Medical, Osaka, Japan). According to the manufacturer, it contains the bifunctional adhesive monomer MDP. Another adhesive system is 5\% 4-META dissolved in methyl methacrylate (MMA) (SuperBond, Sun Medical, Moriyama, Japan). According to the manufacturer, polymerization of SuperBond is initiated by stabilized tri- $n$-butylborane (TBB) in the presence of poly(methyl methacrylate) (PMMA) powder. Favorable results have been reported when silica coating is used in conjunction with bis-GMA-based resin cements, ${ }^{11}$ but to our knowledge, this conditioning system has not been tested with any 4-META-based cement.

The dilemma for clinicians remains whether to follow the manufacturers' instructions for resin cements during cementation of FDPs made of zirconia or to modify the instructions and use different surface conditioning protocols, as suggested by some other manufacturers. Adhesion of the resin cement to zirconia could be less important for FDPs that rely on mechanical retention, ${ }^{16}$ but for less-retentive FDPs, adhesion of resin cements is important for clinical success. ${ }^{17-19}$ This study therefore evaluated the effect of surface conditioning, consisting of silica coating followed by MPS or MPS/4-META silane application, on the adhesion of two resin-based cements to zirconia ceramic in comparison with the conditioning protocols recommended by the manufacturer of each cement. The tested hypotheses were (1) that the silica coating together with the application of silane would result in higher bond strength than the manufacturer-recommended procedures for the evaluated resin cements and (2) that both types of silanes would yield similar results.

\section{Materials and methods}

\section{Specimen preparation}

Disc-shaped (diameter, $15 \mathrm{~mm}$; thickness, $2 \mathrm{~mm}$ ) zirconia ceramics (LAVA, 3M ESPE, Seefeld, Germany) were embedded in polyethylene molds using PMMA (Condular, Wager, Switzerland) with one side of the disc left exposed for cement adhesion (Fig. 1). Specimens were polished with 1200 grit silicone carbide abrasive with water cooling (Struers Rotopol 11, Struers, Rodovre, Denmark) and ultrasonically cleaned in distilled water for $3 \mathrm{~min}$. Subsequently, 60 discs were randomly divided into six groups according to the cement and silane type to be applied ( $n=10$ per group).

Two types of resin cements were selected: a dual-polymerized (Panavia F 2.0) and a chemically polymerized cement (SuperBond). Table 1 summarizes the experimental groups, surface conditioning methods, and the procedures specified by each manufacturer for the resin cements tested, and Table 2 shows the brand names, compositions, batch numbers, and manufacturers of the silane coupling agents and resin cements. 
Both resin cements were mixed and applied in a standardized manner by the same operator using translucent polyethylene molds (inner diameter, $3.6 \mathrm{~mm}$; height, $5 \mathrm{~mm}$ ) attached to the specimen. Each resin cement was mixed according to the manufacturer's instructions and packed into the mold incrementally using a hand instrument. Panavia F 2.0 was photopolymerized with a halogen light polymerization unit (Demetron LC, SDS Kerr, Orange, CA, USA) for $40 \mathrm{~s}$ from a constant distance of $2 \mathrm{~mm}$ from the

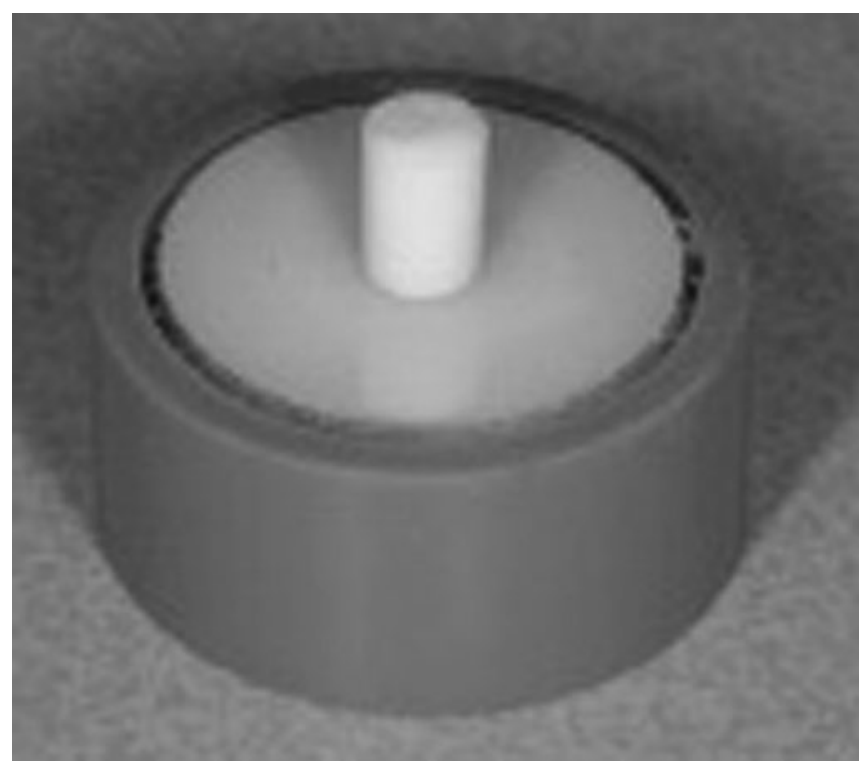

Fig. 1. Yttrium-stabilized tetragonal zirconium polycrystal disc specimen embedded in poly(methyl methacrylic) with the cementation surface exposed. Cement was applied incrementally, not exceeding $2 \mathrm{~mm}$ in thickness, to the ceramic surface in a polyethylene mold (inner diameter, $3.6 \mathrm{~mm}$; height, $5 \mathrm{~mm}$ ) and subsequently polymerized surface under a light intensity of $>450 \mathrm{~mW} / \mathrm{cm}^{2}$, verified by a radiometer (Demetron $\mathrm{LC}$, Kerr). The polyethylene molds were then gently removed, and a polyethylene glycol/glycerin oxygen inhibition gel (Oxyguard II, Kuraray) was applied around the margins of the cement-ceramic interface of the specimens to which Panavia F 2.0 had been applied. These specimens were then further photopolymerized for $40 \mathrm{~s}$. After $3 \mathrm{~min}$, the oxygen inhibition gel was rinsed off thoroughly. All specimens were kept in distilled water at $37^{\circ} \mathrm{C}$ for 3 months in the dark prior to testing.

The specimens were mounted in the jig of the universal testing machine (Zwick ROELL Z2.5 MA 18-1-3/7, Ulm, Germany) and shear loading was applied to the adhesive interface until failure occurred (cross-head speed, $1.0 \mathrm{~mm} /$ $\mathrm{min}$ ). The maximum force required to produce failure was recorded (MPa) using the provided software.

After debonding, the failure sites were examined by two calibrated operators (M.Ö., C.C.) both visually and in digital photographs using a software program (CorelDRAW 9.0, Corel, Ottawa, Canada). Failure was scored as cohesive (three grades) or adhesive (one grade) as follows: 1 , cohesive failure of the cement over $<1 / 3$ of the ceramic surface; 2 , cohesive failure of the cement over $>1 / 3$ of the ceramic surface; 3 , cohesive failure of the ceramic surface; 4 , adhesive failure at the interface with no resin left on the substrate.

\section{Statistical analysis}

Statistical analysis was performed with SAS statistical software (SAS for Windows, release 8.02/200; Cary, NC, USA). The results of uniformity and normality tests indicated that the residual values were normally distributed when plotted against predicted values and thus did not violate the analysis of variance (ANOVA) assumptions. The bond strength data for all

Table 1. Experimental groups, surface conditioning methods, and procedures according to each manufacturer's instructions for the resin cements tested

\begin{tabular}{|c|c|c|c|}
\hline Group & Surface conditioning & Silane coupling agent ${ }^{\mathrm{c}}$ & Resin cement \\
\hline 1 & Silica coating $^{\mathrm{a}}$ & MPS & SuperBond \\
\hline 2 & & MPS/4-META & SuperBond \\
\hline 3 & & MPS & Panavia F 2.0 \\
\hline 4 & & MPS/4-META & Panavia F 2.0 \\
\hline 5 & - & MPS/4-META & SuperBond $^{\mathrm{d}}$ \\
\hline 6 & $\mathrm{Al}_{2} \mathrm{O}_{3}$ air-particle abrasion ${ }^{\mathrm{b}}$ & - & Panavia F $2.0^{\mathrm{e}}$ \\
\hline
\end{tabular}

${ }^{a}$ Chairside silica coating with 30- $\mu \mathrm{m}$ alumina particles coated with silica (CoJet-Sand, 3M ESPE) using an intraoral air-abrasion device (Micro-Etcher, Danville, San Ramon, USA), holding the nozzle perpendicular to the surface from a distance of approximately $10 \mathrm{~mm}$ for $13 \mathrm{~s}$ at a pressure of 2.3 bar. Excess sand was gently blown away with air

${ }^{\mathrm{b}}$ Chairside air-particle abrasion with $50-\mu \mathrm{m}$ alumina particles (Korox, Bego, Bremen, Germany) using the Micro-Etcher intraoral air-abrasion device, holding the nozzle perpendicular to the surface from a distance of approximately $10 \mathrm{~mm}$ for $13 \mathrm{~s}$ at a pressure of $2.3 \mathrm{bar}$. Excess sand was gently blown away with air

${ }^{\mathrm{c}}$ MPS is a one-bottle (ESPE-Sil) and MPS/4-META is a two-bottle silane system (Porcelain Liner M) mixed in a 1:1 ratio. See Table 2 for the compositions of each. The silane solutions were applied to the ceramic substrate in a thin layer. A new brush was used for each application and they were allowed to react for $15 \mathrm{~min}$ at room temperature. The manufacturer of Porcelain Liner $M$ recommends applying heat to the silanized surfaces for improving the bond strength. Heat was applied with a small hair dryer to each specimen silanized with MPS/4-META for 2 min

${ }^{\mathrm{d}}$ Manufacturer's instructions: Apply MPS/4-META coupling agent only

${ }^{\mathrm{e}}$ Manufacturer's instructions: Apply air-particle abrasion only 
Table 2. Brand name, composition, batch numbers, and manufacturers of the silane coupling agents and resin cements

\begin{tabular}{llll}
\hline Brand & Composition & Batch number & Manufacturer \\
\hline ESPE-Sil & MPS, ethanol & 68411 & 3M ESPE, Seefeld, Germany \\
Porcelain Liner M & Liquid A: & MT1 & Sun Medical, Moriyama, Japan \\
& 4-META/MMA & & \\
& Liquid B: & & Sun Medical \\
SPS/MMA & 4-META/MMA-TBB & MT3 & \\
& Type: Chemical polymerized & & \\
& Initiator: TBB derivative & & Kuraray Medical, Osaka, Japan \\
& Monomer liquid: 5 wt\% & & \\
4-META/MMA & Polymer powder (clear): pulverized PMMA & & \\
& Type: Dual-polymerized & 41144 & \\
& MDP & & \\
& Paste A: BPEDMA/MDP/DMA & & \\
& Paste B: Al-Ba-B-Si glass/silica- containing composite & & \\
\hline
\end{tabular}

4-META, 4-methacryloyloxyethyl trimellitate anhydride; MMA, methyl methacrylate; MPS, 3-methacryloxypropyltrimethoxysilane; TBB, tri- $n$ butylborane; PMMA, poly(methyl methacrylate); MDP, 10-methacryloxydecyldihydrogen-phosphate; BREDMA, bisphenol-A-polyethoxydmethacrylate; DMA, dimethacrylate

Fig. 2. Shear-bond strengths $(\mathrm{MPa})$ of the experimental groups. Box plots represent, from bottom to top, the minimum value, first quartile, median, third quartile, and maximum value. See Table 1 for group definitions

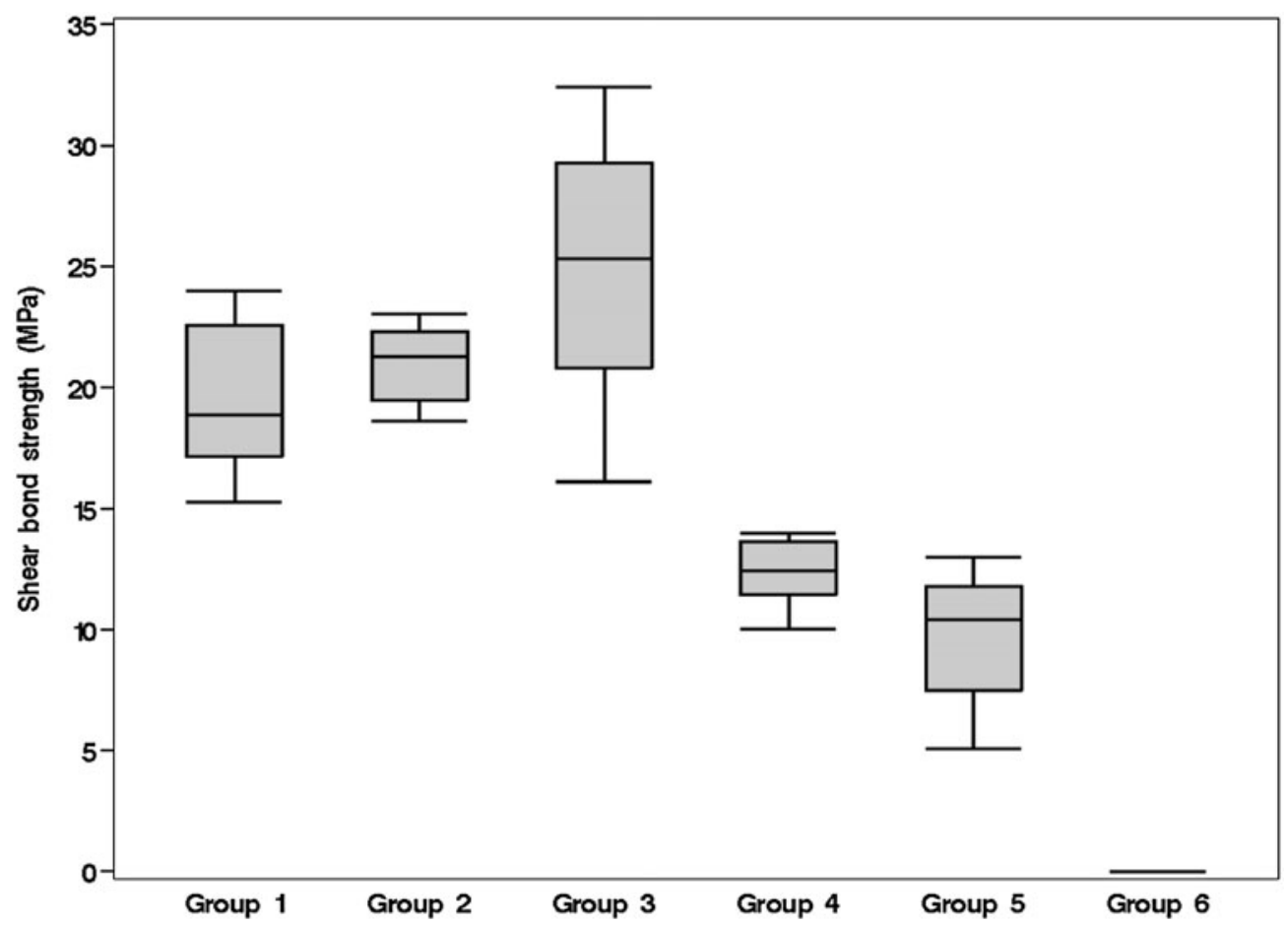

groups were analyzed by one-way ANOVA, considering each group as one adhesion protocol. Owing to the significant difference among the groups $(P<0.001)$, multiple comparisons were made using Tukey's HSD adjustment test. To study the effect of silane and cement type, two-way ANOVA and Tukey's HSD test were used for groups 1-4 with mean bond strength being the dependent variable. $P$ values less than 0.05 were considered to be statistically significant in all tests.

\section{Results}

When the ceramic surfaces were silica coated and SuperBond cement was used, mean bond strength was not signifi- cantly different between MPS and MPS/4-META silanes (groups 1 and 2, mean $\pm \mathrm{SD}, 20.2 \pm 3.7$ and 20.9 $\pm 1.6 \mathrm{MPa}$, respectively). However, when Panavia F 2.0 cement was used, mean bond strength was significantly higher with MPS silane (group 3, $24.4 \pm 5.3 \mathrm{MPa}$ ) than with MPS/4-META silane (group 4, $12.3 \pm 1.4 \mathrm{MPa})(P<0.001$; ANOVA, Tukey's test) (Fig. 2).

When silica coating and silanization were employed, both Panavia F 2.0 and SuperBond cements demonstrated higher bond strengths results than when they were not. When the manufacturer's instructions were followed for each cement, the mean bond strength with SuperBond cement (group 5, 9.73 $\pm 1 \mathrm{MPa}$ ) was significantly higher than that with Panavia F 2.0 (group 6, $0 \mathrm{MPa})(P<0.001)$. As 
described in the Methods, Panavia F 2.0 was applied to the ceramic surface by using a transparent polyethylene mold and then polymerized, but when the mold was removed, the cement came off with the mold. Thus, Panavia F 2.0 was not able to bond to the ceramic surface at all, and no bond strength value could be measured.

In groups 5 and 6, failure was exclusively adhesive $(100 \%)$ (score 4). Failure in groups 1-4 was cohesive, with $70 \%, 70 \%, 70 \%, 60 \%$, respectively, of specimens receiving a failure score of 2 and the remaining specimens in each group receiving a score of 1 .

\section{Discussion}

It has been previously demonstrated that the bond strength of resin-based materials bonded to acid-resistant ceramics, especially to zirconia, is neither durable nor stable, presenting a challenge to clinicians, especially when bonding nonretentive zirconia FDPs. ${ }^{20,21}$ In this study, when silica coating and silanization were employed for surface conditioning, both 4-META/MMA-TBB- and MDP-based cements demonstrated higher bond strengths than they did when the manufacturers' instructions were followed. Therefore, our first hypothesis was confirmed. The silica coating in this study was applied with a chairside air abrasion device. Airparticle abrasion seems to be a prerequisite for achieving the improved bond strength between the studied resins and high-strength ceramics that are reinforced with either alumina or zirconia. ${ }^{7}$ Air abrasion of the surface generates hydroxyl groups on the surface and enhances micromechanical retention. Furthermore, the methoxy groups of silane react with water to form silanol groups, which in turn react with the surface hydroxyl groups to form a siloxane network. In principle, the presence of a ceramic glassy phase favors formation of siloxane bonds, because the silanol groups to react with the silica to form a siloxane (-Si-O-SiO-) network on the surface. Since no silica phase exists in zirconia ceramics, the silica coating on the ceramic surface presumably provides a substrate for reaction with the silane, thus enhancing the adhesion of the silane layer and thereby the adhesion of the luting cements.

When applied following silica coating, MPS silane performed better with Panavia F 2.0 than the MPS/4-META silane; hence, our second hypothesis is not accepted. This different performance can be explained if MPS silane improved the wettability and surface energy properties. However, interestingly, there were no significant differences between MPS and MPS/4-META silanes with SuperBond cement when the ceramic surfaces were silica coated. This result may be due to the better compatibility of 4-META/ MMA-TBB with the methacrylate groups of the two silanes tested. Further chemical analysis is in progress in our laboratories. The adhesion of the resins to the ceramic substrates seems to depend on the presence of silica on the surface compatible with the silane coupling agent, as better results were obtained with these procedures than when the manufacturers' instructions only were followed. In ceramic-resin adhesion, silane functions as a coupling agent, which by adsorbing onto and altering the surface of the ceramic, facilitates chemical interaction. The silane coupling agent is rapidly hydrolyzed in the presence of an acidic monomer such as MDP. After being hydrolyzed, the silane coupling agent can react with hydroxyl groups on the surface silica and form siloxane bonds. SuperBond does not contain a functional acidic monomer such as MDP. In this case, polysiloxane formation with a strong molecular structure is promoted by the acidity of the substrate surface treated with a silane coupling agent. ${ }^{22}$ Nevertheless, both adhesive cements benefited from preliminary surface conditioning by silica coating. This result suggests that the performance of the tested cements on zirconia can be improved by air-abrasion conditioning prior to silane application.

Air abrasion systems rely on air-particle abrasion with

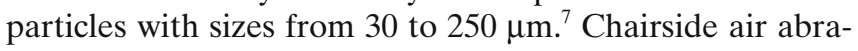
sion is considered less aggressive with respect to the zirconia material itself and also with the restoration margins because a smaller particle size and lower pressure is used than when air abrasion is performed in the laboratory. ${ }^{10}$ The abrasion process removes loose, contaminated layers, and the roughened surface allows some degree of mechanical interlocking with the adhesive. Air abrasion may also introduce physicochemical changes that affect surface energy and wettability. Cement-ceramic adhesion, however, is susceptible to chemical, thermal, and mechanical influences under intraoral conditions. One limitation of this study is that we did not conduct thermocycling, although the effect of thermocycling is controversial. ${ }^{11,23,24}$ Some earlier studies reported high and stable bond strength to zirconia after airborne particle abrasion using $\mathrm{Al}_{2} \mathrm{O}_{3}$ particles in combination with an MDP-based resin cement. ${ }^{23,24}$ It has been hypothesized that the chemical adhesion between aluminum oxides and silanes presents a higher potential for hydrolytic degradation than that between silicone oxide and silanes. ${ }^{25} \mathrm{~A}$ recent study, however, reported inferior results with Panavia F-zirconia adhesion, with a dramatic decrease in performance after 12000 thermocycles or long-term (300 days) water storage. ${ }^{11}$ Although reactions may have occurred between hydroxyl groups in the MDP monomer and hydroxyl groups on the zirconia ceramic surface, the chemical bonds thus formed did not maintain their strength after thermocycling. Therefore, hydrolytic stability of these material combinations should be further investigated under different aging conditions. Nonetheless, this study provides important data regarding cementation-related early clinical failures with zirconia FPDs. ${ }^{16-19}$

There are also some recent developments in the field of zirconate coupling agents. ${ }^{25}$ Zirconate coupling agents might slightly bond with hydroxyl groups on a zirconia surface, but a significant decrease in bond strength was observed after thermocycling, depending on the silane concentration. It is evident that silane coupling agents with better chemical affinity to zirconium oxides that are at the same time hydrolytically more stable are needed. The phosphate ester group of MDP has been reported to bond directly to metal oxides such as chromium, nickel, aluminum, tin, titanium, and zirconium oxides. $^{23,26}$ The findings of the present study suggest that an 
MDP monomer may not bond chemically to zirconia without surface conditioning and silanization.

Material selection and clinical recommendations on resin bonding are based on laboratory tests that show great variability in materials and methods. One of the most common testing methods is the shear bond test. However, in microtensile tests, stress distribution is reported to be more homogeneous. ${ }^{27}$ Therefore, the results of this study should be verified by microtensile testing, especially since practically no adhesion was achieved when Panavia was applied according to the manufacturer's instructions, with the zirconia air-abraded with alumina particles only. Instead, the polyethylene molds filled with this cement completely detached from the zirconia surface when the mold was removed. These results, recorded as a bond strength of $0 \mathrm{MPa}$, were a worst-case scenario. One can argue whether they should be interpreted as premature failure, but previous studies have recorded debondings experienced during cutting procedures for microtensile tests or during water storage or thermocycling as a bond strength of $0 \mathrm{MPa} \cdot{ }^{9-11}$ Unfortunately, there is no consensus in the literature on dental materials regarding this. In future studies, use of materials other than polyethylene for the molds should be considered as well. Nevertheless, probably there was no interaction between the mold and the Panavia F 2.0 cement because in the other groups, this problem was not experienced. The results clearly indicate that the interfacial strength in group 6 did not exceed even the frictional forces between the cement and the mold.

\section{Conclusions}

The results of this study suggest the following conclusions:

1. When silica coating and silanization were used, both SuperBond and Panavia F 2.0 cements demonstrated higher bond strengths than they did when the manufacturers' instructions were followed.

2. When the ceramic surfaces were silica coated, there was no significant difference between MPS and MPS/4META silanes with SuperBond cement, but with Panavia F 2.0, when MPS silane was used, bond strengths were significantly higher than when MPS/4-META was used.

Acknowledgments We acknowledge R. Rutgers for his assistance during the experimental procedures.

\section{References}

1. Piconi C, Maccauro G. Zirconia as a ceramic biomaterial. Biomaterials 1999;20:1-25.

2. Guazzato M, Albakry M, Ringer SP, Swain MV. Strength, fracture toughness and microstructure of a selection of all-ceramic materials Part II. Zirconia-based dental ceramics. Dent Mater 2004;20:449-56.

3. Luthardt RG, Sandkuhl O, Reitz B. Zirconia-TZP and aluminaadvanced technologies for the manufacturing of single crowns. Eur J Prosthodont Restor Dent 1999;7:113-9.

4. Ardlin BI. Transformation-toughened zirconia for dental inlays, crowns and bridges: chemical stability and effect of low-temperature aging on flexural strength and surface structure. Dent Mater 2002;18:590-5.
5. Drummond JL. Ceramic behavior under different environmental and loading conditions. In: Eliades G, Eliades T, Brantley WA, Watts DC. Dental materials in vivo: Aging and related phenomena. Carol Stream: Quintessence; 2003. p. 35-45.

6. Blatz MB, Sadan A, Kern M. Resin-ceramic bonding: a review of the literature: J Prosthet Dent 2003;89:268-74.

7. Özcan M, Alkumru HN, Gemalmaz D. The effect of surface treatment on the shear bond strength of luting cement to a glass-infiltrated alumina ceramic. Int J Prosthodont 2001;14:335-9.

8. Özcan M, Vallittu PK. Effect of surface conditioning methods on the bond strength of luting cement to ceramics. Dent Mater 2003;19:725-31.

9. Amaral R, Bottino MA, Valandro LF, Özcan M. Microtensile bond strength of a resin cement to glass infiltrated zirconia-reinforced ceramic: the effect of surface conditioning. Dent Mater 2006;22: 283-90.

10. Valandro LF, Özcan M, Bottino MC, Scotti R, Bottino MA, Della Bona A. Bond strength of a resin cement to high-alumina and zirconia-reinforced ceramics: the effect of surface conditioning. $\mathrm{J}$ Adhes Dent 2006;8:175-81.

11. Valandro LF, Özcan M, Amaral R, Leite FP, Bottino MA. Microtensile bond strength of a resin cement to silica-coated and silanized In-Ceram Zirconia before and after aging. Int $\mathrm{J}$ Prosthodont 2007;20:70-2.

12. Zhang Y, Pajares A, Lawn BR. Fatigue and damage tolerance of Y-TZP ceramics in layered biomechanical systems. J Biomed Mater Res B Appl Biomater 2004;71:166-71.

13. Plueddemann EP. Adhesion through silane coupling agents. J Adhes 1970;2:184-201.

14. Ooij van WJ, Sabata A. Characterization of films of organofunctional silanes byTOF SIMS and XPS.Part I.Films of $N$-(2-(vinylbenzylamino) ethyl)-3-aminopropyltrimethoxysilane on zinc and 3-aminopropyltriethoxysilane on steel substrates. In: Mittal KL, editor. Silanes and other coupling agents. Utrecht: VSP, 1992; p. 323-43.

15. Mohsen NM, Craig RG. Hydrolytic stability of silanated zirconia silica-urethane dimethacrylate composites. J Oral Rehabil 1995;22: 213-20.

16. Edelhoff D, Özcan M. To what extend does the longevity of fixed dental prostheses depend on the function of cement? Clin Oral Implants Res 2007:18:193-204.

17. Raigrodski AJ, Chiche GJ, Potiket N, Hochstedler JL, Mohamed SE, Biliot S, Mercante DE. The efficacy of posterior three-unit zirconium-oxide-based ceramic fixed partial dental prostheses: a prospective clinical pilot study. J Prosthet Dent 2006:96:237-44.

18. Sailer I, Feher A, Filser F, Luthy H, Gauckler LJ, Scharer P, Hammerle CF. Prospective clinical study of zirconia posterior fixed partial dentures: 3-year follow-up. Quintessence Int 2006;37: 685-93.

19. Wassermann A, Kaiser M, Strub JR. Clinical long-term results of VITA In-Ceram Classic crowns and fixed partial dentures: a systematic literature review. Int J Prosthodont 2006;19:355-63.

20. Dérand P, Dérand T. Bond strength of luting cements to zirconium oxide ceramics. Int J Prosthodont 2000;13:131-5.

21. Tinschert J, Zwez D, Marx R, Anusavice KJ. Structural reliability of alumina-, feldspar-, leucite-, mica- and zirconia-based ceramics. J Dent 2000;28:529-35.

22. Miller JD, Hoh KP, Ishida H. Studies of the simulation of silane coupling agent structures on particulate fillers; the $\mathrm{pH}$ effect. Polym Composites 1984;5:18-28.

23. Kern M, Wegner SM. Bonding to zirconia ceramic: adhesion methods and their durability. Dent Mater 1998;14:64-71.

24. Wegner SM, Kern M. Long-term resin bond strength to zirconia ceramic. J Adhes Dent 2000;2:139-47.

25. Yoshida K, Tsuo Y, Atsuta M. Bonding of dual-cured resin cement to zirconia ceramic using phosphate acid ester monomer and zirconate coupler. J Biomed Mater Res B Appl Biomater 2006;77:28-33.

26. Wada T. Development of a new adhesive material and its properties. In: Gettleman L, Vrijhoef MMA, Uchiyama Y, editors. Proc Int Symp Adhes Prosthodont, Amsterdam, Netherlands. Chicago: Academy of Dental Materials; 1986. p. 9-18.

27. Della Bona A, van Noort R. Shear vs. tensile bond strength of resin composite bonded to ceramic. J Dent Res 1995;74:1591-6. 